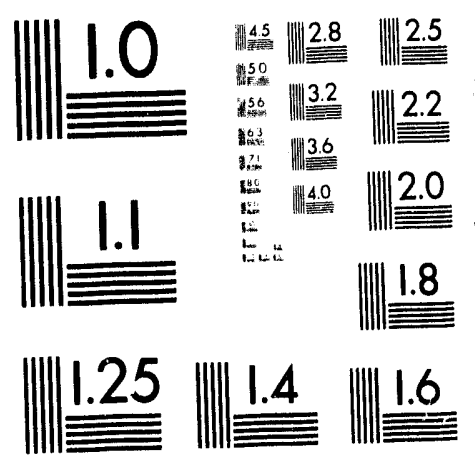



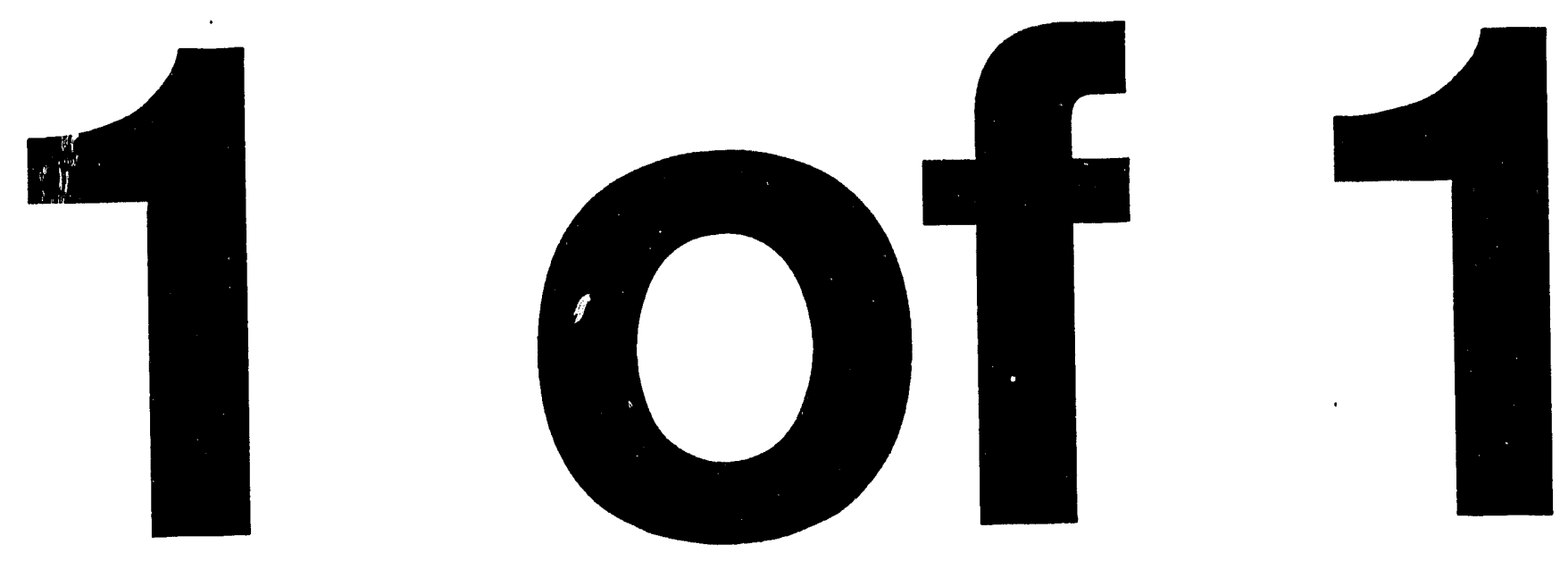
UCK.L- JC-114779

PREPRINT

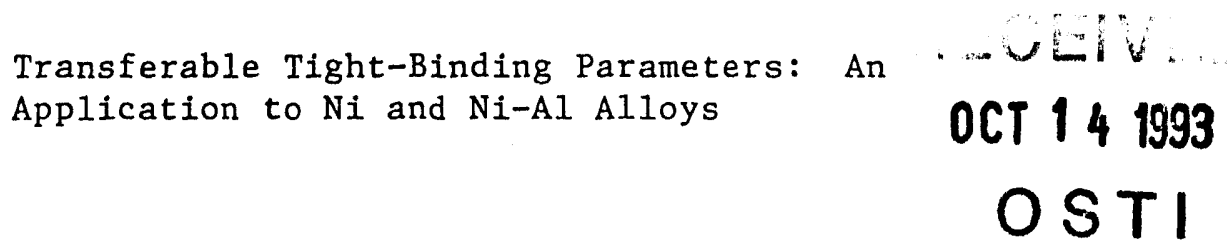

Marcel H. F. Sluiter and Prabhakar P. Singh

This paper was prepared for submittal to the NATO Advanced Research Workshop, Metallic Alloys: Experimental and Theoretical Perspectives, Boca Raton, Florida, July 16-21, 1993.

This is a preprint of a paper intended for publication in a journal or proceedings. Since changes may be made before publication, this preprint is made available with the understanding that it will not be cited or reproduced without the permission of the author. 
DISCLAIMER

This docomeat was prepared as a accouat of work spoasored by an aseacy of the Inited States Goverament. Weither tbe Laited States Goverament wor the Luiversity of Californis nor an! of theif employees. makes any warnany, express or inplied. or assumes an! kegal liability or responsibility for the eccuracy. completeness. or usefulness of any iaformation. apparatus. produce of process disclosed or represeats that its use would sol iafriace privately onned rights. Refereace serein to any specific comraercial products, process or service by trade anme, trademart, manofactarer. or otherwise does wor meressarily constifnte or imply its endorsemeah recoenaeadation. or taroring by the L'ailed States Coverameat or the Lairersity of Califonia The views and opinions of atbors expressed berein do sot acesssarily state er reflect those of the U'uited Sules Governmeat or the Uaiversity of Califorain, and stall oof be ased for advertising or product eadorsement purposes. 


\title{
TRANSFERABLE TIGHT-BINDING PARAMETERS: AN APPLICATION TO NI AND NI-AL ALLOYS
}

\author{
MARCEL H.F. SLUITER and PRABHAKAR P. SINGH \\ Lawrence Livermore National Laboratory, L-268 \\ P.O. Box 808, Livermore, CA 94550, USA
}

\begin{abstract}
Two approaches for obtaining tight-binding parameters for metallic alloys are compared and contrasted with special regard for the application to large scale simulations such as may occur in tight-binding molecular dynamics studies.
\end{abstract}

\section{Introduction}

Modeling of materials processes and properties is becoming more and more important in the field of materials science. Many properties pertaining to perfectly crystalline ordered materials can be computed with high accuracy from electronic structure methods. Properties such as lattice parameters, elastic constants and heats of formation for pure elemental metals and ordered in termetallic compounds are examples. However, many important physical properties are critically dependent on extended defects in crystalline or configurational order, such as e.g. mechanical behavior, which is dictated largely by the generation and mobility of dislocations, and electrical conductivity, which is dependent on the state of short- or long range order. These defects which may exist only in low concentrations in an otherwise nearly perfect crystal pose a serious challenge to the com putational materials scientist.

Electronic structure methods based on the local density approximation which are used so successfully for the study of small periodic unit cells become unwieldy when large numbers of atoms must be considered. It is here that elegant parametrizations of the electronic structure, such as the one provided by the tight-binding (TB) method, have great appeal. Such a parametrization reduces the computational effort by orders of magnitude but at the expense of accuracy and knowledge of the spatial charge-distribution. Currently there are two approaches for obtaining tight-binding parameters that accurately reflect the electronic structure of metals and alloys;

1) Fitting. Energy-eigenvalues at various points in the Brillouin zone (BZ) are computed with a highly accurate first-principles method. The TB parameters are regarded as adjustable parameters which are selected such that the energy-eigenvalues are reproduced as accurately as possible. Such Slater-Koster (SK) parameters for the pure elements have been published by Papaconstantopoulos [1].

2) The linear muffin-tin orbital (LMTO) Hamiltonian can be transformed into a tight-binding (TB) form. Andersen and collaborators [2] have shown that when muffin-tin orbitals are screened with multipoles on neighboring sites, the screened orbitals have an almost crystal structure independent decay in space. Invoking the atomic sphere approximation allows the TB formulation to be cast in its most convenient two-center form. The screened orbitals can be used to define (structure dependent) canonical two-center TB integrals which can be made element specific through the conventional LMTO potential parameters. 
TB parametrizations must meet :ieveral conditions in order to describe the electronic structure of the distorted atomic configurations that occur in actual materials:

1) parameters must be valid for a wide range of atorilic coordinations and environments,

2) parameters must apply over a range of in teratomic separations,

3) chemical effects between unlike atomic species must be accurately described.

The extent to which these requirements are met will be examined below. I will regard the LMTO and the Korringa-Kohn-Rostoker coherent potential approximation (KKR-CPA) [3] results as "exact".

Nickel-Aluminum alloys were selected for this study because extensive first-principles electronic structure calculations are available for this alloy, and because as an alloy of a transition metal and a normal metal it is not a priori obvious that a TB approach can provide an accurate description.

\section{Results and Discussion}

LMTO calculations were performed on $\mathrm{Ni}$ with the fcc, bcc and A15 crystal structures at a Wigner-Seitz (WS) radius of 2.60 a.u. These three structures were selected because they reflect a wide variety of atomic environments. FCC is close-packed and has 12 fold atomic coordination. BCC is not-so close-packed but still is a simple structure and has 14-fold atomic coordination. A 15 is among the simplest complex structures, and atoms have either 12- or 14-fold coordinations. The potential parameters of relevance to the $T B$ parametrization are given in table 1.

Table 1. LMTO Potential Parameters (in Ry) of $\mathrm{Ni}$ with the fcc, bcc and A15 crystalline structures.

\begin{tabular}{|l|ccrcr|}
\hline & T & $\mathrm{E}_{v}$ & \multicolumn{1}{c}{$\mathrm{C}$} & \multicolumn{1}{c|}{$\Delta$} & \multicolumn{1}{c|}{$\gamma$} \\
\hline fcc & 0 & -0.4711 & -0.3180 & 0.1862 & 0.4265 \\
& 1 & -0.3118 & 0.7343 & 0.1739 & 0.1135 \\
& 2 & -0.2104 & -0.1821 & 0.0119 & -0.0025 \\
\hline bcc & 0 & -0.4707 & -0.3185 & 0.1858 & 0.4265 \\
& 1 & -0.3133 & 0.7323 & 0.1736 & 0.1135 \\
& 2 & -0.2096 & -0.1815 & 0.0119 & -0.0026 \\
\hline A15 & 0 & -0.4806 & -0.3207 & 0.1863 & 0.4267 \\
& 1 & -0.3207 & 0.7325 & 0.1741 & 0.1135 \\
& 2 & -0.2164 & -0.1887 & 0.0119 & -0.0025 \\
\hline
\end{tabular}

It is clear that the variation in atomic environment has little influence on the potential parameters. As a consequence, when these potential parameters are used to compute the SK parameters of say fcc Ni only small variations are found, typically less than about $4 \mathrm{mRy}$ in the onsite energies and less than 0.5 $\mathrm{mRy}$ in the hopping parameters (see table 2). This means that SK parameters for complicated structures with large unit cells can be computed with potential parameters from LMTO calculations on much simpler structures. 
Table 2. Slater-Koster Parameters in fcc paramagnetic Ni obtained from A15 (1), bcc (2), and fcc (3) lmto potential parameters and (4) as obtained from the fit to APW results by D.A. Papaconstantopoulos [1]. To facilitate the comparison the fitted onsite energies have been shifted rigidly so as to $m$ atch the TB-LMTO fcc $t_{2 g}$ onsite energy.

\begin{tabular}{|c|c|c|c|c|c|}
\hline fcc & SK & (1) & (2) & (3) & (4) \\
\hline$[000]$ & $\begin{array}{l}s \\
p \\
t_{2 g} \\
e_{g}\end{array}$ & $\begin{array}{r}0.163082 \\
0.538837 \\
-0.157983 \\
-0.166814\end{array}$ & $\begin{array}{r}0.168288 \\
0.544057 \\
-0.150625 \\
-0.159480\end{array}$ & $\begin{array}{r}0.169437 \\
0.546883 \\
-0.151158 \\
-0.160033\end{array}$ & $\begin{array}{r}0.44342 \\
0.89403 \\
-0.15116 \\
-0.15431\end{array}$ \\
\hline $1 / 2$ a [110] & $\begin{array}{l}s s \sigma \\
\operatorname{pp} \sigma \\
\mathrm{pp} \pi \\
\mathrm{dd} \sigma \\
\mathrm{dd} \pi \\
\mathrm{dd} \delta \\
s p \sigma \\
\text { sd } \sigma \\
\operatorname{pd} \sigma \\
\operatorname{pd} \pi\end{array}$ & $\begin{array}{r}-0.078474 \\
0.139982 \\
-0.017498 \\
-0.041885 \\
0.017915 \\
-0.001640 \\
0.104397 \\
-0.055178 \\
-0.074883 \\
0.017829\end{array}$ & $\begin{array}{r}-0.078795 \\
0.140426 \\
-0.017553 \\
-0.041997 \\
0.017963 \\
-0.001644 \\
0.104776 \\
-0.055364 \\
-0.075102 \\
0.017881\end{array}$ & $\begin{array}{r}-0.078905 \\
0.140846 \\
-0.017606 \\
-0.042092 \\
0.018003 \\
-0.001648 \\
0.105005 \\
-0.055465 \\
-0.075299 \\
0.017928\end{array}$ & $\begin{array}{r}-0.09525 \\
0.21708 \\
0.01660 \\
-0.03712 \\
0.02629 \\
-0.00600 \\
0.14003 \\
-0.03880 \\
-0.04400 \\
0.02377\end{array}$ \\
\hline a [100] & $\begin{array}{l}s s \sigma \\
\text { pp } \sigma \\
\text { pp } \pi \\
\text { dd } \sigma \\
\text { dd } \pi \\
\text { dd } \delta \\
\text { sp } \sigma \\
\text { sd } \sigma \\
\operatorname{pd} \sigma \\
\operatorname{pd} \pi\end{array}$ & $\begin{array}{r}-0.003243 \\
0.006299 \\
0.000000 \\
-0.002902 \\
-0.000252 \\
0.000000 \\
0.004261 \\
-0.002714 \\
-0.004160 \\
-0.000297\end{array}$ & $\begin{array}{r}-0.003256 \\
0.006319 \\
0.000000 \\
-0.002909 \\
-0.000253 \\
0.000000 \\
0.004277 \\
-0.002723 \\
-0.004172 \\
-0.000298\end{array}$ & $\begin{array}{r}-0.003261 \\
0.006338 \\
0.000000 \\
-0.002916 \\
-0.000254 \\
0.000000 \\
0.004286 \\
-0.002728 \\
-0.004183 \\
-0.000299\end{array}$ & $\begin{array}{r}-0.00065 \\
0.06220 \\
0.00682 \\
-0.00651 \\
0.00344 \\
-0.00027 \\
0.01441 \\
-0.01015 \\
-0.01012 \\
0.00510\end{array}$ \\
\hline
\end{tabular}

There are some noticeable differences between the TB-LMTO and the fitted SK parameters. The $s$ and $p$ onsite energies are much farther above the d onsite energies in the fitted SK parameters. Moreover, some hopping parameters have significantly different values or even different signs (pp $\pi$ in $1^{\text {st }}$ shell, $\mathrm{dd} \pi$ and $\mathrm{pd} \pi$ in $2^{\text {nd }}$ shell). It appears that the higher $s$ and $\mathrm{p}$ onsite energies in the fit are compensated for by larger hopping parameters. To some extent the larger hopping parameters in the fit result from the smaller WS radius $(2.56$ a.u.).

It is well known that SK parameters depend on interatomic distance. The most common relationship employed to take this distance scaling into account is the one derived by Andersen et al [4] from the LMTO formalism:

$$
\beta_{I r M}(d)=\beta_{I r M}\left(d_{o}\right) \cdot\left(\frac{d}{d_{o}}\right)^{l+r+1} \text {. }
$$

where $\beta$ represents a hopping parameter and $d$ represents distance between the 

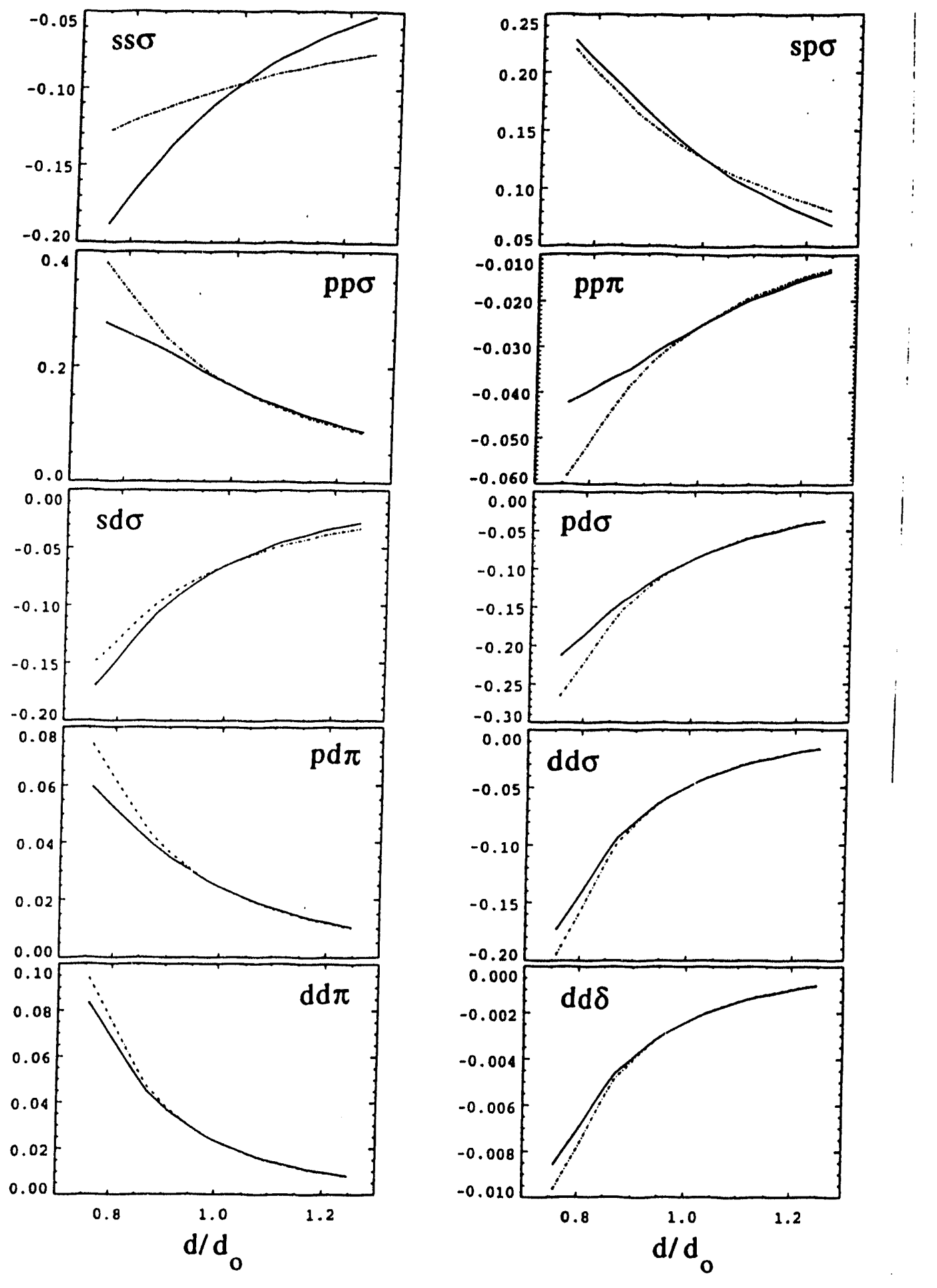

Figure 1. Hopping parameters in the nearest neighbor shell in fcc $\mathrm{Ni}$ as a function of interatomic distance as computed with eq. (1) (dashed line) and as computed directly from the LMTO potential parameters (solid line). 
two nuclei. The validity of this expression was evaluated by performing LMTO calculations on fcc $\mathrm{Ni}$ at various lattice parameters and using the potential parameters to obtain SK parameters. These directly calculated SK parameters were compared with those extrapolated from the equilibrium lattice parameters using eq. (1), the results are displayed in figure 1 .

As expected, eq. (1) is especially accurate in the atomic limit (large $d / d_{o}$ ) and for large angular momentum $l+l$. In previous work [5] it was concluded that eq. (1) is valid for up to about $5 \%$ interatomic distance changes. Here, we find that the expression holds for essentially arbitrarily large expansions but breaks down at more than about 10 percent compression.

Usually, the change of the onsite energies with the WS radius is ignored. Here, it is found that the onsite energies can shift significantly with respect to each other, see figure 2. Therefore, when the WS radius changes not only the hopping parameters but also the onsite energies must be adjusted. This can be conveniently accomplished with in the TB-LMTO method by means of the potential parameter derivatives listed in table VII of reference [2].

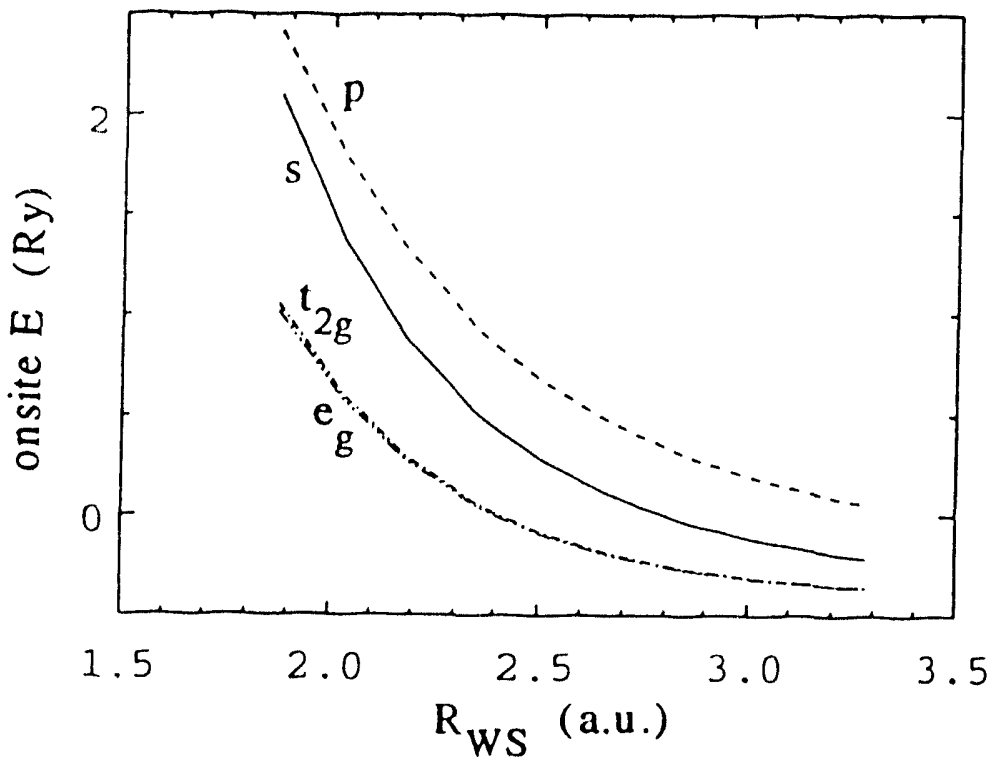

Figure 2. Onsite energies in $\mathrm{fcc} \mathrm{Ni}$ as a function of $\mathrm{WS}$ radius as computed from the LMTO potential parameters.

It is by no means obvious that TB parameters obtained from pure elements would still be applicable within a concentrated alloy. As a check the LMTO potential parameters of both $\mathrm{Ni}$ and $\mathrm{Al}$ were computed for the pure fcc elements and for the $\mathrm{B} 2 \mathrm{NiAl}$ intermetallic compound, results are listed in table 3. Clearly, at the same WS radii the potential parameters from the pure element and from the $\mathrm{B} 2 \mathrm{NiAl}$ calculation differ significantly. The differences are much reduced when the $\mathrm{B} 2 \mathrm{NiAl}$ potential parameters are evaluated in the vicinity of its own equilibrium WS radius. Nevertheless, it is clear that the potential parameters are much more sensitive to the chemical environment than to the crystalline structure. 
Table 3. LMTO Potential Parameters (in Ry) of Ni as obtained from LMTO calculations on the fcc pure element and the $\mathrm{B} 2 \mathrm{NiAl}$ intermetallic compound. FCC: $R_{w s}=2.60$ a.u., $B 2(1): R_{w s}=2.60$ a.u., $B 2(2): R_{w s}=2.683$ a.u.

\begin{tabular}{|lccrcr|}
\hline & & $\mathrm{E}_{v}$ & \multicolumn{1}{c}{$\mathrm{C}$} & $\Delta$ & \multicolumn{1}{c|}{$\gamma$} \\
\hline FCC & 0 & -0.4711 & -0.3180 & 0.1862 & 0.4265 \\
& 1 & -0.3118 & 0.7343 & 0.1739 & 0.1135 \\
& 2 & -0.2104 & -0.1821 & 0.0119 & -0.0025 \\
\hline B2 (1) & 0 & -0.4151 & -0.2825 & 0.1899 & 0.4266 \\
& 1 & -0.2448 & 0.7707 & 0.1761 & 0.1134 \\
& 2 & -0.1117 & -0.0745 & 0.0131 & -0.0036 \\
\hline B2 (2) & 0 & -0.4405 & -0.3256 & 0.1736 & 0.4250 \\
& 1 & -0.2853 & $0.6 \times 32$ & 0.1625 & 0.1128 \\
& 2 & -0.1516 & -0.1187 & 0.0113 & -0.0037 \\
\hline
\end{tabular}

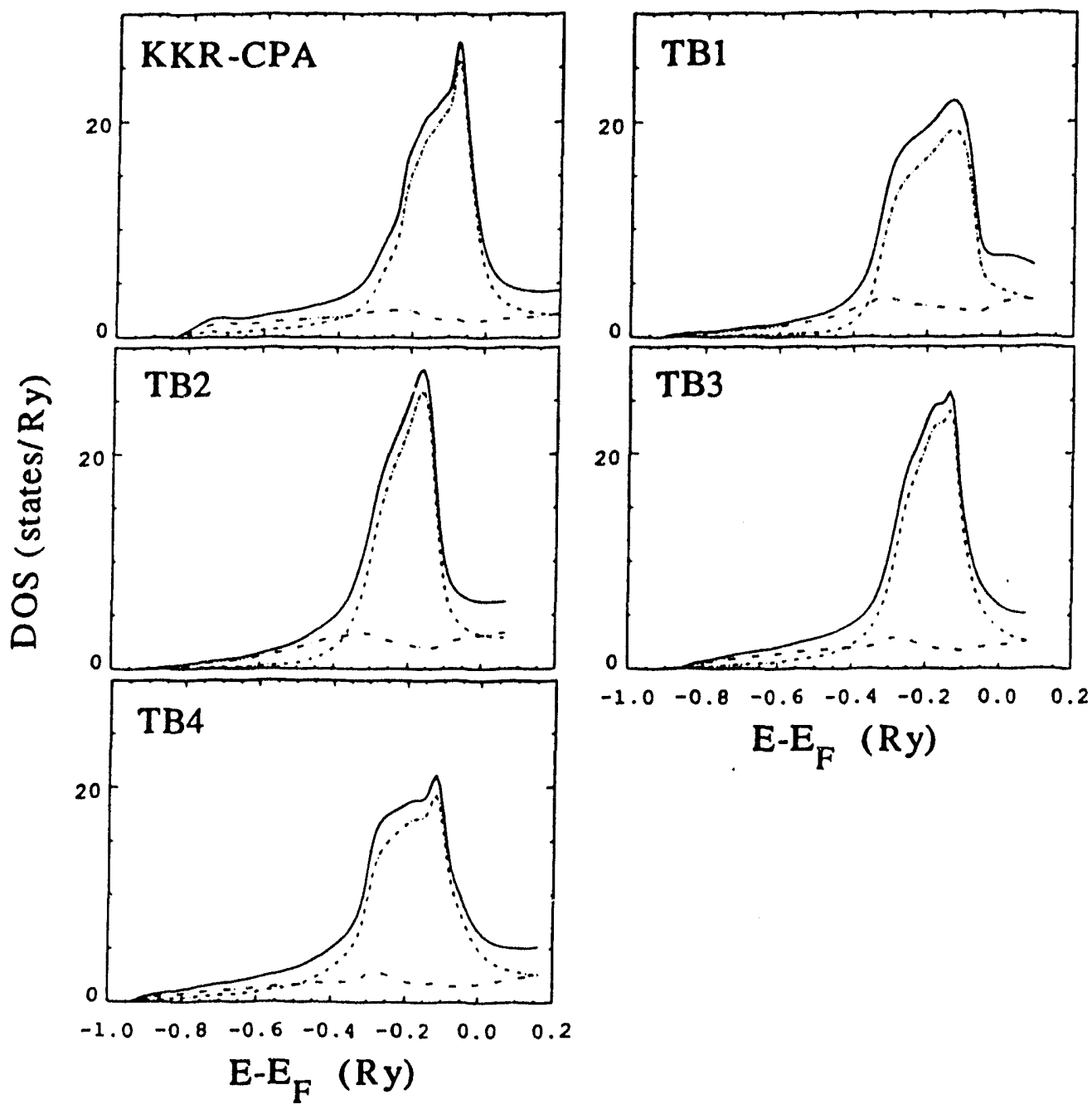

Figure 3. DOS of $\mathrm{fcc} \mathrm{Ni}_{0.5} \mathrm{Al}_{0.5}$ as obtained with the KKR-CPA and various TB approximations (see text). Total DOS (solid line), Ni partial DOS (dash-dotted line), and Al partial DOS (dash triple-dotted line). 
Another verification of the applicability of the various TB parameters has been performed by calculating the DOS of the fcc equiatom ic alloys with the CPA. The TB results are compared with a KKR-CPA result in figure 3 . The DOS marked TB1 has been computed using the parameter for fcc $\mathrm{Al}$ and fcc paramagnetic $\mathrm{Ni}$ listed in [1] without any modifications. In comparison with the KKR-CPA results, the bandwidth is much larger and the Ni d-band appears too low. These shortcomings can be somewhat mitigated by scaling the hopping parameters according to eq. (1) for changes in the WS radius, and by rigidly shifting the $\mathrm{Ni}$ onsite energies down by $100 \mathrm{mRy}$ with respect to the $\mathrm{Al}$ onsite energies. The results thus obtained are marked TB2.

It must be noted that a rigid shift of the onsite energies of one element generally is required because the data in [1] are given with reference to the muffin-tin zero which is different for each element, structure and WS radius. The TB2 result has a slightly too narrow Ni d-band which is still somewhat too far below the Fermi-level and, related, there are too few states in the bottom of the band.

In TB3 the TB-LMTO was used with SK parameters from the pure elements rescaled with eq (1). for the change in the WS radius. The Ni d-band is too narrow and too far below the Fermi-level. It appears that the characteristic feature of the sharp peak at the upper edge of the $\mathrm{Ni} d$-band is correctly described. The SK parameters derived from the B2 NiAl structure give rise to the DOS marked TB4. Here, the d-band is again too wide but otherwise the agreement with the KKR-CPA is fairly good.

It is rather difficult to evaluate which TB DOS best approximates the KKR-CPA result and hence examining the DOS may not be very discriminating.

A more critical test is provided by the effective pair interactions (EPI) as defined by the generalized perturbation method (GPM) [6]. The EPI indicate the presence and nature of ordering or clustering in an alloy.

Equiatomic $\mathrm{Ni}$-Al alloys form an extremely stable bcc based ordered intermetallic compound with the $\mathrm{B} 2$ ( $\mathrm{CsCl}$ type) structure. The occurrence of this phase implies that the nearest neighbor EPI is strongly positive as has indeed been found in KKR-CPA-GPM calculations [7].

The question whether the various TB approaches correctly describe the nature of the chemical interactions (EPI) in this alloy has been addressed in figure 4. It is clear that the SK parameters from [1] do not at all describe the EPI in the actual Ni-Al system as the computed values have the opposite sign. Moreover, the parameters cannot be adjusted by shifting the onsite energies of one element with respect to another, or by judiciously applying eq. (1) such that a positive nearest neighbor EPI comes about.

The TB-LMTO approaches successfully describe the variation of the EPI with the e/ a ratio. The SK parameters derived from the B2 NiAl structure give somewhat less accurate EPI values than those obtained from the pure element TB-LMTO derived SK parameters. The latter give an excellent agreement with the KKR-CPA-GPM result. The poorer performance by the B2 NiAl derived SK parameters is probably due to the strong ionicity in B2 NiAl which is carried over by the SK parameters into the actually not so ionic disordered bcc. The excellent agreement between TB-LMTO-CPA-GPM and KKR-CPA-GPM results is found for the whole composition range and extends to the angular momentum decomposed EPI, as is shown in figure 5. 

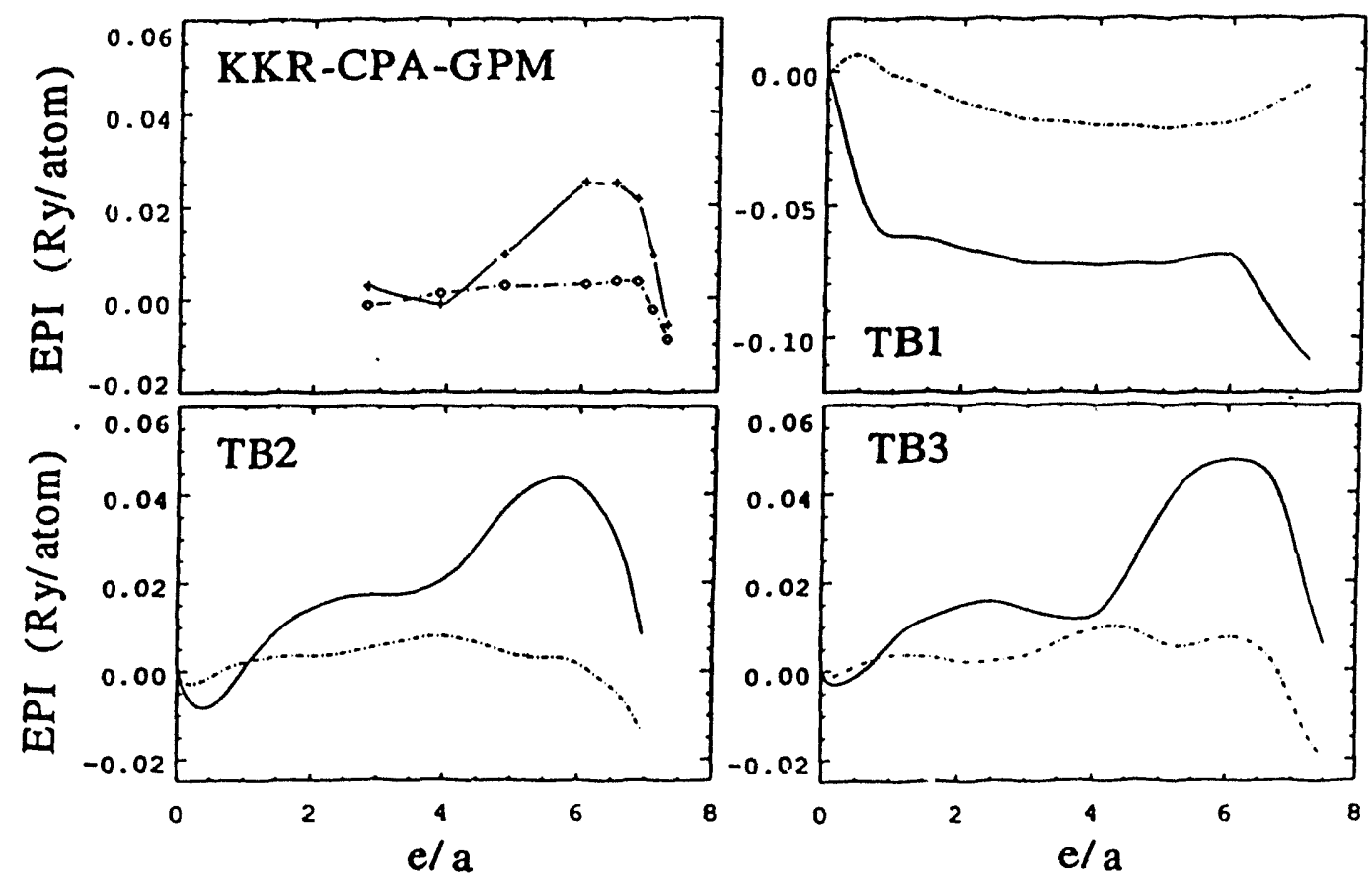

Figure 4. $1^{\text {st }}$ (solid line) and $2^{\text {nd }}$ nearest neighbor EPI (dashed line) in bcc $\mathrm{Ni}_{0.5} \mathrm{Al}_{0,5}$ as a function of the electron/atom ratio as computed with the KKR$C P A$ with the frozen potential approximation, and as computed with various TB parametrizations. TB1: SK parameters from [1], TB2: TB-LMTO with potential parameters from fcc $\mathrm{Ni}$ and fcc AI, TB3: TB-LMTO with potential parameters from B2 NiAl.

The agreement between the KKR-CPA-GPM and the TB-LMTO-CPA-GPM results is remarkable. Clearly, the TB-LMTO describes the chemical interaction between $\mathrm{Ni}$ and $\mathrm{Al}$ in much detail.

The composition dependence of the EPI also is described well by the TBLMTO as is shown in figure 6 . Only towards high $\mathrm{Ni}$ concentration does a noticeable discrepancy exist. The TB-LMTO-CPA-GPM predicts that the $1^{\text {st }}$ nearest neighbor EPI keeps increasing with $\mathrm{Ni}$ concentration, whereas the KKR-CPA-GPM predicts that the $1^{\text {st }}$ nearest neighbor EPI becomes essentially composition independent at high $\mathrm{Ni}$ content.

\section{Conclusion}

It has been shown that the SK parameters obtained from the TB-LMTO formalism have a number of advantages over those obtained from highly precise fits to electronic bands. These advantages are: (1) The TB-LMTO potential parameters are structure independent and hence one LMTO calculation can yield SK parameters for a wide range of crystal structures. (2) The TB-LMTO SK parameters observed the scaling law (eq. 1) fairly well, and the onsite energies too can be adjusted easily when the WS radius changes. 

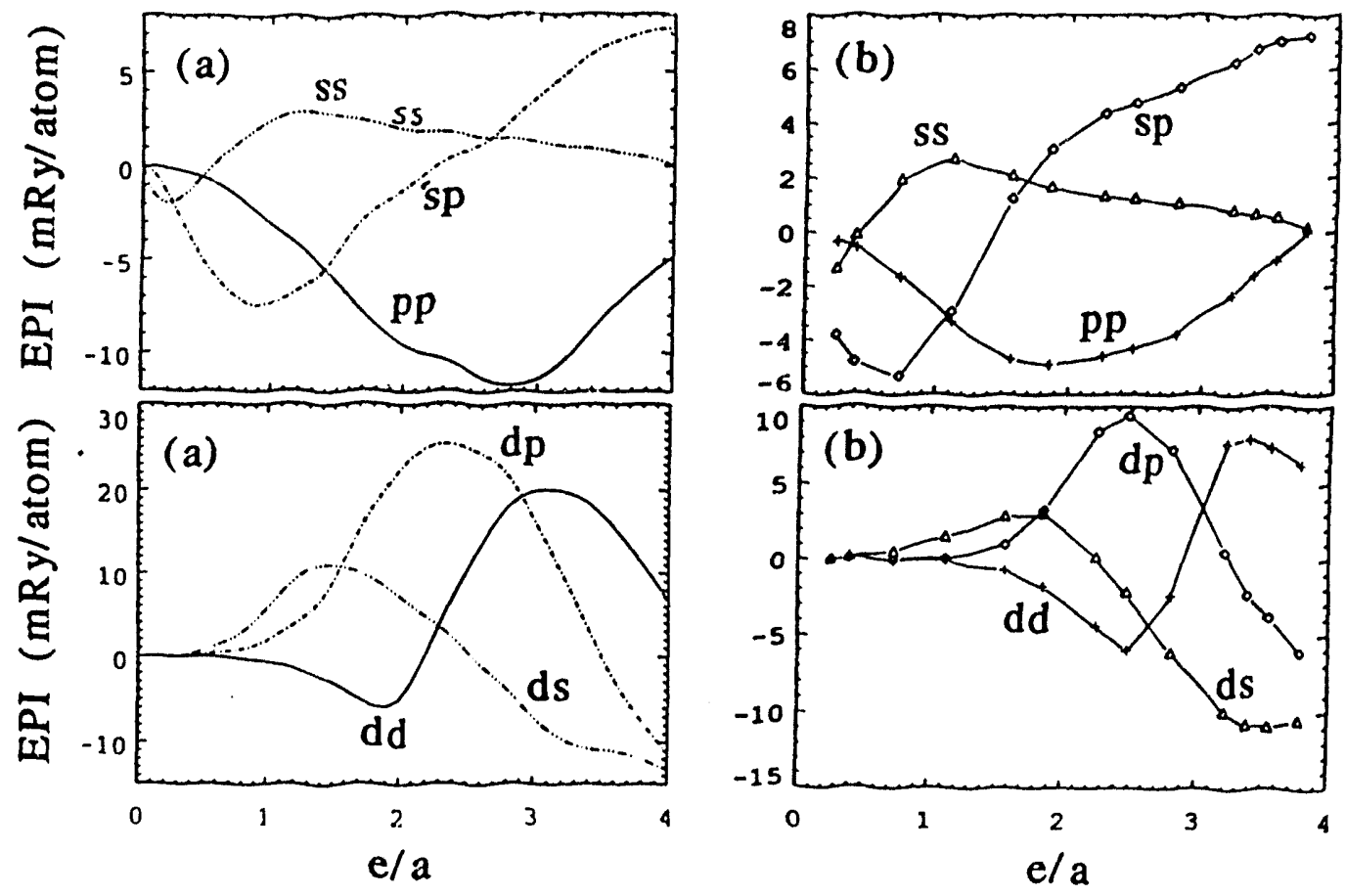

Figure 5. The angular momentum decomposed nearest neighbor EPI in fcc $\mathrm{Ni}_{0,2} \mathrm{Al}_{0.2}$ as a function of electron per atom ratio as computed with (a) TBLMTO-CPA-GPM (using potential parameters from pure fcc elements) and (b) KKR-CPA-GPM.

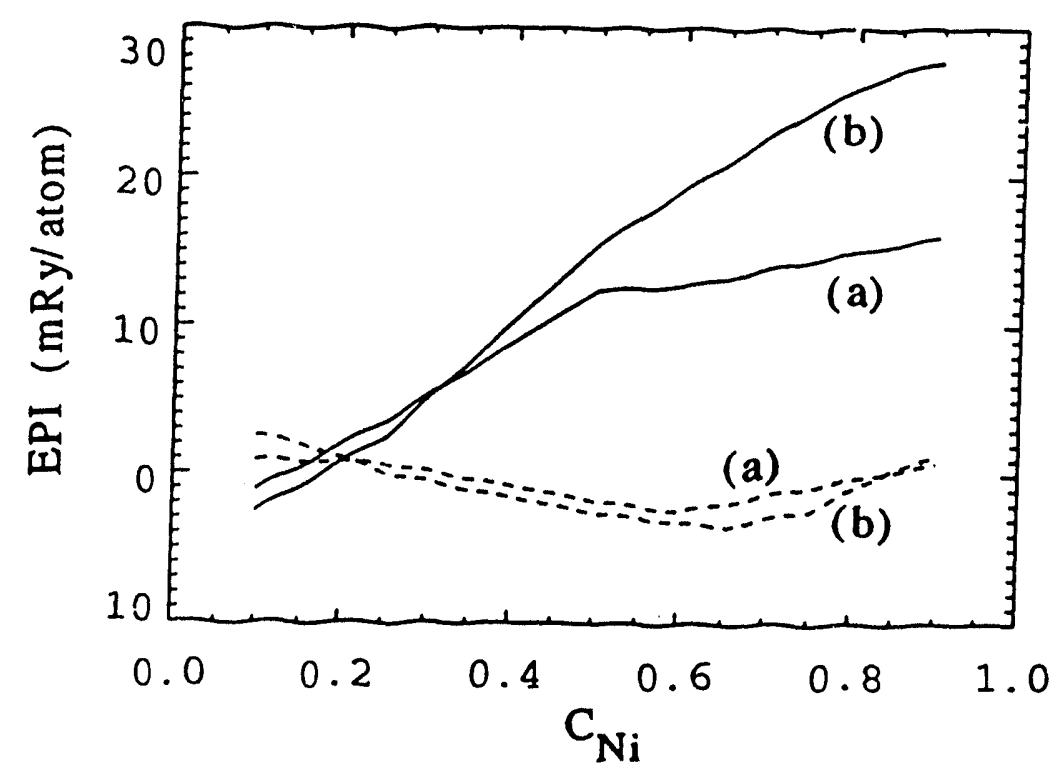

Figure 6. The $1^{\text {st }}$ (solid line) and $2^{\text {nd }}$ nearest neighbor EPI (dashed line) in fcc $\mathrm{Ni}$-Al alloys as a function of composition as computed with (a) KKR-CPAGPM and (b) TB-LMTO-CPA-GPM (using potential parameters from pure fcc elements). 
(3) Unlike the fitted SK parameters, the TB-LMTO SK parameters are defined with reference to the Coulomb potential so that no arbitrary rigid shift of the onsite energies is needed when alloys are considered. (4) The TB-LMTO SK parameters describe the chemical interactions with remarkable accuracy, unlike the fitted SK parameters. It must be noted however, that the DOS of an alloy is described just as well by the fitted SK parameters as by the TB-LMTO. It is concluded that for simulations such as TB-molecular dynamics simulations the TB-LMTO approach is the one of choice.

This work was performed under the auspices of the U.S. Department of Energy by the Lawrence Livermore National Laboratory under contract no. W-7405ENG-48.

\section{References}

[1] D.A. Papaconstantopoulos, "Handbook of the Band Structure Elemental Solids", (Plenum, New York, 1986).

[2] O.K. Andersen, O. Jepsen, and D. Glotzel, in "Highlights of Condensed Matter Theory", Internat. School of Phys. Enrico Fermi, Course 89, eds. F. Bassani, F. Fermi, and M.P. Tosi, 59, (North Holland, Amsterdam, 1985).

[3] J. S. Faulkner, in "Progress in Materials Science", ed. by J. W. Christian, P. Haasen, and T. B. Massalski (Pergam on Press, New York, 1982), Vol. 27 , p. 1; and references cited therein.

[4] O.K. Andersen, W. Klose, and H. Noh!, Phys. Rev. E 17, 1209 (1978).

[5] J.D. Shore and D.A. Papaconstantopoulos, Phys. Rev. B 35, 1122 (1987).

[6] F. Ducastelle, in "Alloy Phase Stability", NATO ASI Series E: Applied Sciences Vol. 163, ed. by G.M. Stocks and A. Gonis, (Kluwer Academic Publishers, Boston, 1989), p. 293; and references cited therein.

[7] M. Sluiter, P.E.A. Turchi, F.J. Pinski, and G.M. Stocks, Journal of Phase Equilibria 13, 605 (1992). 

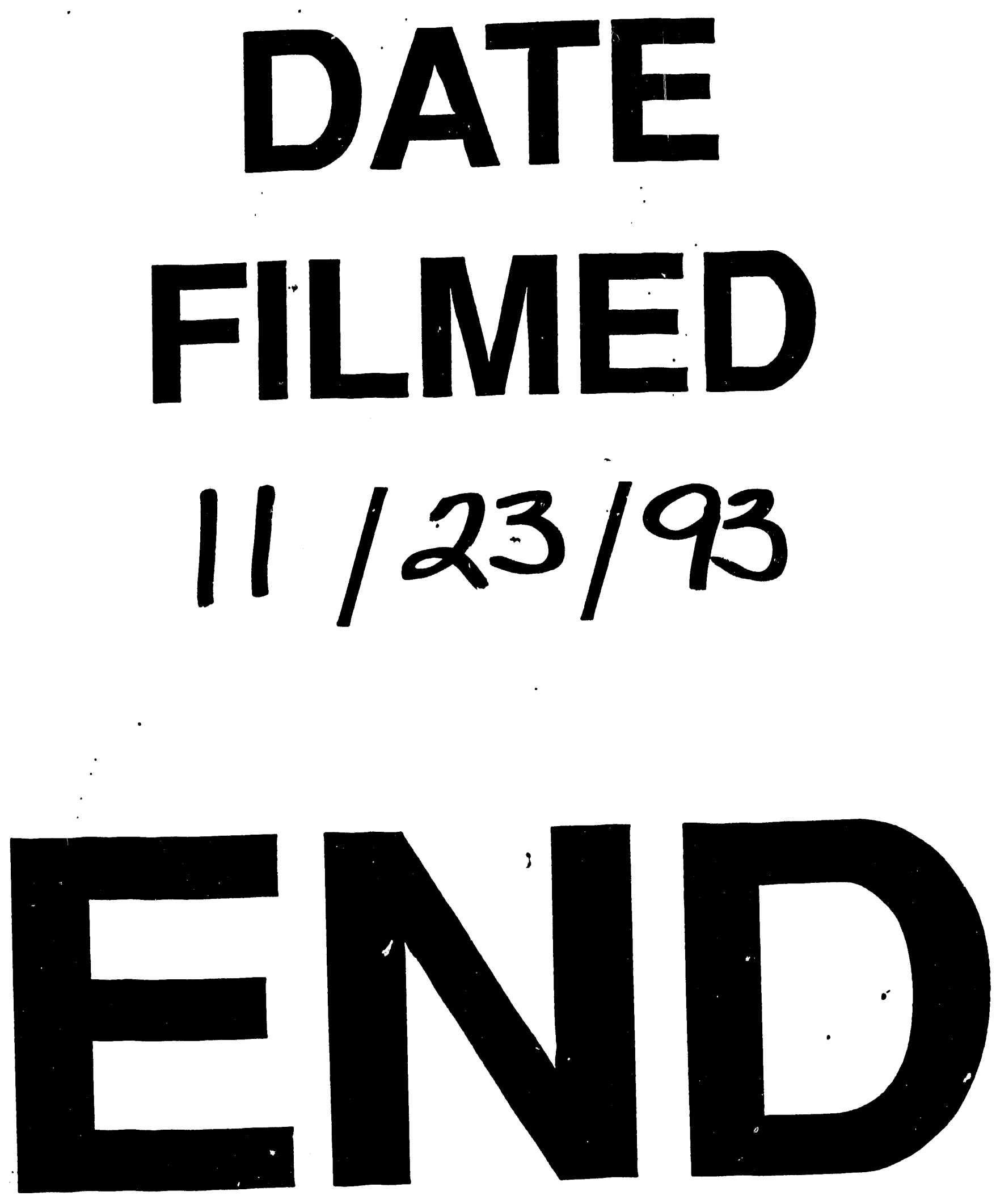
